\title{
A Performance Analysis of Folding Conformal Propeller Blade Designs
}

\author{
Brandon L. Litherland* and Joseph M. Derlaga ${ }^{\dagger}$ \\ NASA Langley Research Center, Hampton, VA, 23681, U.S.A.
}

\begin{abstract}
NASA's X-57 Maxwell flight demonstrator has a high-lift system that includes 12 fixedpitch high-lift propellers located upstream of the wing leading edge for lift augmentation at low speeds. These high-lift propellers are only required at low speeds, and to reduce drag, the propeller blades are folded conformally along the nacelles at other operating conditions. The method of designing the high-lift blades permits several variations of blade cross-section placement along the nacelle surface and a comparative performance analysis was needed to determine if any particular design showed significant benefits. We analyzed the performance of three conformal high-lift propeller designs and compared them to that of a non-conformal baseline propeller to establish both the benefit of stowable blades and the value of each variation. In this study, we first performed a drag analysis of each design in the stowed configuration at the $\mathrm{X}-57$ cruise speed and altitude to determine the drag benefits of each conforming method. Then, among blade designs we compared the thrust, power, and lift for a given input shaft speed to establish any performance losses from the baseline. This analysis shows that the conformal blade designs do not have any appreciable performance losses compared to the baseline blades. Moreover, although the drag in the cruise condition is significantly less than for the non-folding baseline, the drag benefits of each conforming blade approach are similar and the value of each approach largely depends on the ease of integration into the nacelle. This paper presents the results of these studies and discusses the benefits and drawbacks of implementing the conformal blade designs. Specifically, we demonstrate that folding, conformal propeller blades contribute significantly less to cruise drag when compared to windmilling, with an increase relative to a clean nacelle of only $3 \%$ versus $81 \%$. We also show a less than $1 \%$ difference in performance between conformal, folding propellers and the non-conforming baseline propeller.
\end{abstract}

\section{Introduction and Background}

This paper presents background, design and analysis methods, and results for a performance study of variations in folding, conformal propeller designs for the X-57 Maxwell flight demonstrator generated using the method presented in 2017 by Litherland et al. [1]. The X-57 Maxwell has 12 high-lift nacelles, each with a 5-bladed high-lift propeller. A high-lift propeller design is required that minimally contributes to drag in the cruise configuration while also maintaining desired performance in the high-lift configuration. The 2017 paper showed that there was a slight, but acceptable, decrease to both thrust and power with conformal blades compared to a non-conformal baseline. We then hypothesized that by spinning the propeller at a slightly higher speed, these differences could be mitigated.

Our intent in this study is to demonstrate that folding, conformal propellers can greatly reduce drag versus a non-folding, windmilling propeller and that the performance is relatively unaffected by modifying the blade for stowage. We will also discuss the trade between design complexity and drag and the reasoning behind choosing one design over another.

\section{A. X-57 Maxwell}

The X-57 Maxwell, NASA's first manned X-plane developed in two decades, is a fully electric flight demonstrator vehicle intended to show that distributed electric propulsion technology can dramatically increase the cruise efficiency over the baseline aircraft [2]. This improvement is achieved by sizing the wing for cruise rather than for takeoff and landing and integrating propellers in a way that takes advantage of flow characteristics at relevant speeds. Figure 1 illustrates the relative sizes of the original wing and the cruise-optimized X-57 wing. The smaller, cruise-optimized $\mathrm{X}-57$ wing would normally require significantly higher takeoff and approach speeds, but by using a distributed electric propulsion (DEP) high-lift propeller system in combination with flaps, the aircraft can achieve the same stall speed 
as the baseline aircraft. The "high-lift propellers" are so named because they are designed to act as high-lift devices at low speeds, rather than as traditional propulsors, by accelerating the air over the wing thus increasing the local dynamic pressure and lift. At higher velocities, the lift augmentation is no longer needed and the high-lift propellers are ineffective. At these speeds, the high-lift propellers are unpowered and would normally result in a large drag contribution if left deployed and windmilling. Instead, the X-57 high-lift propeller blades fold back into a stowed configuration and are designed such that they conform to the attached nacelles. The goal of the X-57 Maxwell is to demonstrate a $5 \mathrm{x}$ improvement in cruise efficiency over the baseline aircraft by combining the increased efficiency of electric motors versus internal combustion engines with the effects of the cruise-optimized wing and the aero-propulsive integration benefits of using tip propellers. Conformally folding the high-lift propellers supports the cruise efficiency goal by lessening the drag contribution of each nacelle.

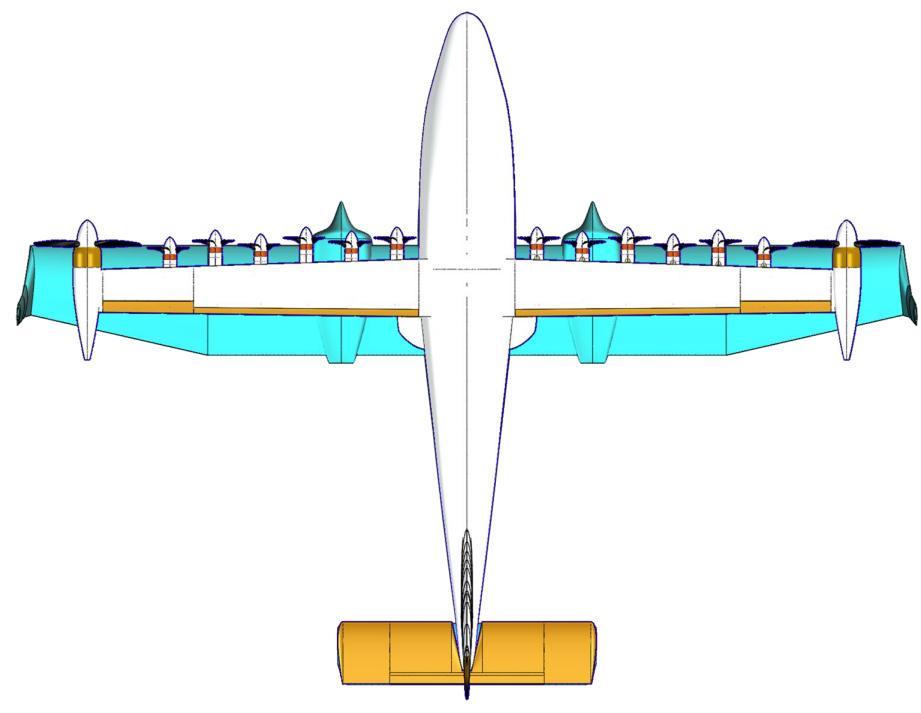

Fig. 1 Comparison of the X-57 Maxwell flight demonstrator over the baseline Tecnam P2006T aircraft (cyan). The X-57's distributed electric propulsion wing is $42 \%$ of the P2006T wing area, enabling more efficient flight at higher speeds.

\section{B. Folding Propeller Blades}

A folding propeller blade's purpose is to provide thrust at various required flight conditions while lessening the propeller drag by stowing the blades when the propeller is not operating, usually by aligning the blade length with the freestream flow. There are several examples of folding propellers in aeronautics, typically included in powered gliders such as the Alisport Silent 2 Electro [3] and the Stemme S-10 [4]. Additional examples include: remote controlled aircraft propellers [5], DEP or urban air mobility (UAM) research aircraft [6-8], and sailboat auxiliary propulsion [9].

The X-57 high-lift nacelles are too small to incorporate complex blade folding mechanisms and the weight constraints are such that feathering hardware cannot be included in the design. However, the nacelles are too big to simply allow the blades to fold directly back and protrude from the surface. Furthermore, the cruise efficiency is sensitive to flow disturbances over the wing and the project determined that the blades must be able to stow. Therefore, the X-57 high-lift propulsion system necessitated a novel, systematic approach to designing folding propeller blades that enabled a simple, single-axis folding mechanism, conformed the blades to the nacelle to minimize drag, and sacrificed no appreciable performance relative to the non-folding blade design.

\section{Baseline Propeller}

The baseline high-lift blades were designed to produce a relatively constant axial velocity profile downstream of the propeller for lift augmentation along the wing [10]. This design set the chord and twist distributions for the conformal 
blades and provided the performance constraints and goals for future designs. The constant-velocity blade was designed for performance at the low tip speed operating point without any consideration for folding. Given the difficulty of including pitch change mechanisms in the relatively small high-lift nacelles, the propellers were designed as fixed-pitch, which results in reasonable performance at low forward flight speeds.

Table 1 provides the baseline, non-conformal high-lift propeller design parameters and conditions. Fig. 2 shows a single baseline blade alongside one of the conformal variants for comparison. In this image, the front view is defined as the view from the front of the aircraft looking aftward and the top view is the view from above the aircraft looking downward. An OVERFLOW analysis indicated that the baseline propeller produces $221 \mathrm{~N}$ of thrust and requires 10.6 $\mathrm{kW}$ of input shaft power at a speed of $4550 \mathrm{RPM}$, closely matching the expected performance.

\section{Table 1 Baseline High-Lift Propeller Specifications}

\begin{tabular}{l|c|l|c} 
Parameter & Value & Parameter & Value \\
\hline Diameter & $22.67 \mathrm{in.}$ & Speed & $4549 \mathrm{RPM}$ \\
Radius & $11.34 \mathrm{in.}$ & Power & $10.5 \mathrm{~kW}$ \\
3/4 Twist & $27.4^{\circ}$ & Thrust & $220 \mathrm{~N}$ \\
No. Blades & 5 & Flight Velocity & 58 KTAS, Sea Level
\end{tabular}
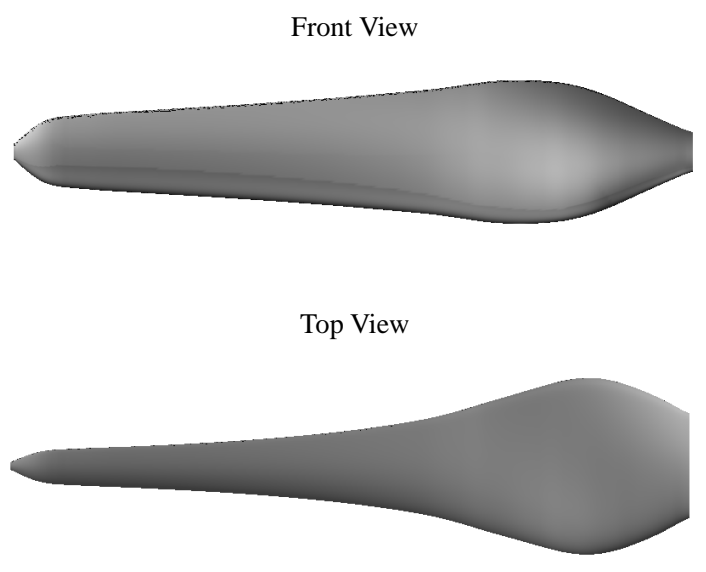

(a) Non-Conformal Blade
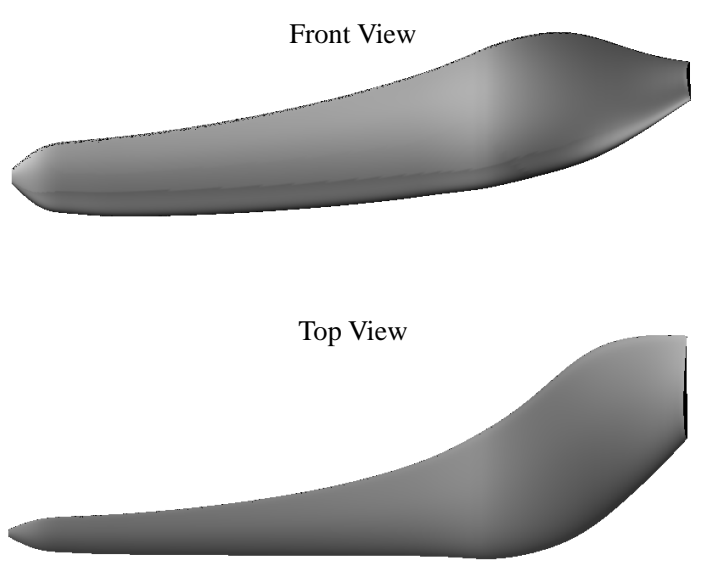

(b) Conformal Blade

Fig. 2 Profile views of the baseline, non-conformal blade along with a modified, conformal blade.

\section{Blade Design Variants}

We produced three blade designs for this study: a fully recessed blade, fully external blade, and a blade designed for a larger diameter spinner. In each of the blade variants, the blade root is recessed completely into the nacelle to smooth the transition between the nacelle and blade surfaces, as shown in Fig. 3. 


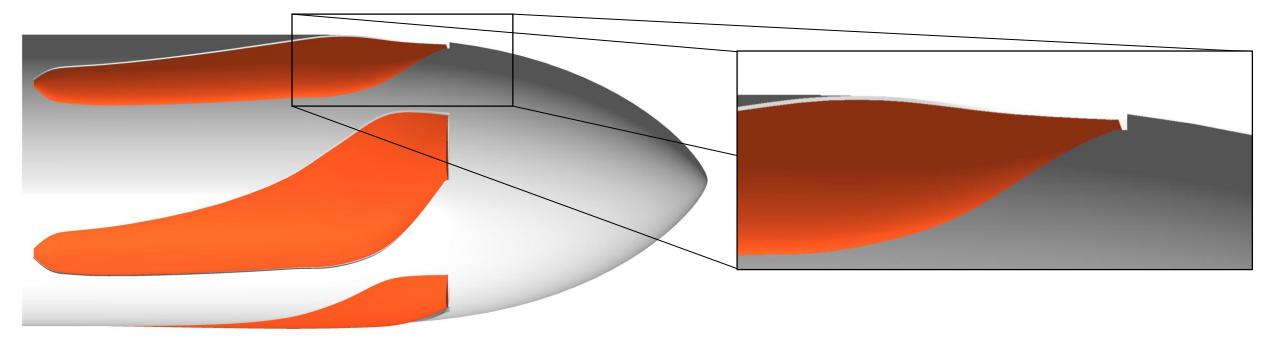

Fig. 3 CAD representation of the smooth transition between the nacelle and blade surfaces. Fully recessed design shown.

\section{A. Fully Recessed Blade}

The fully recessed blade design (Fig. 4) is motivated by the heuristic that the parasitic drag resulting from the exposed wetted surface area should be minimized. Therefore, in this design the entire length of the blade is recessed into the nacelle surface. To ensure that the blades always fold back into a recession, the propeller must stop at certain locations when unpowered, or the recessions must rotate with the propeller. Stopping the blades in this way would require that either a mechanical apparatus or motor controller algorithm be implemented to restrict where and how the propeller comes to rest. For the fully recessed design, the maximum nacelle outer diameter (OD) is 6.36 inches.
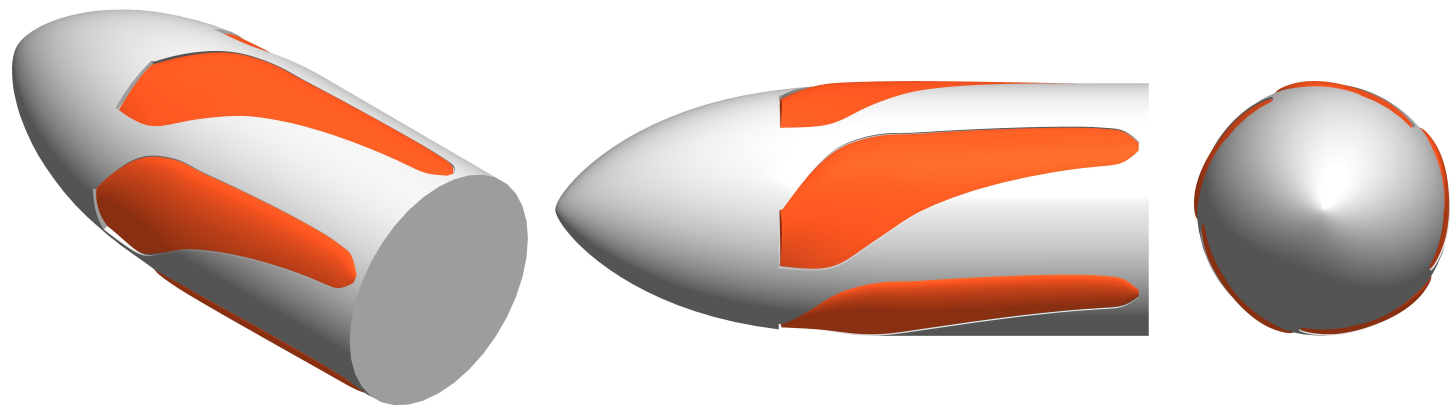

Fig. 4 CAD representation of the fully recessed blade configuration.

\section{B. Larger Diameter Spinner}

Designing for a larger diameter spinner allows the stowed blade region aft of the spinner to simply skim along the nacelle surfaces as shown in Fig. 5, as opposed to requiring recesses cut into the nacelle. In this way, the propeller does not have to be stopped in any particular position. Furthermore, each nacelle section aft of the spinner may be designed as a simple, smooth, cylindrical shell, which reduces the manufacturing and installation complexity. Not cutting into the nacelle also reduces the likelihood of stress concentrations in the nacelle and further reduces risk to project schedule and cost by simplifying many parts. This design has slightly more frontal area and wetted surface area than the fully recessed baseline, which will likely increase the drag versus the recessed configuration. In this design, the maximum spinner OD is increased to 6.94 in., while maintaining the nacelle OD of 6.36 in. resulting in a 0.29 in. step diameter reduction behind the spinner, which will likely cause flow recirculation and increased drag over the recessed design. 

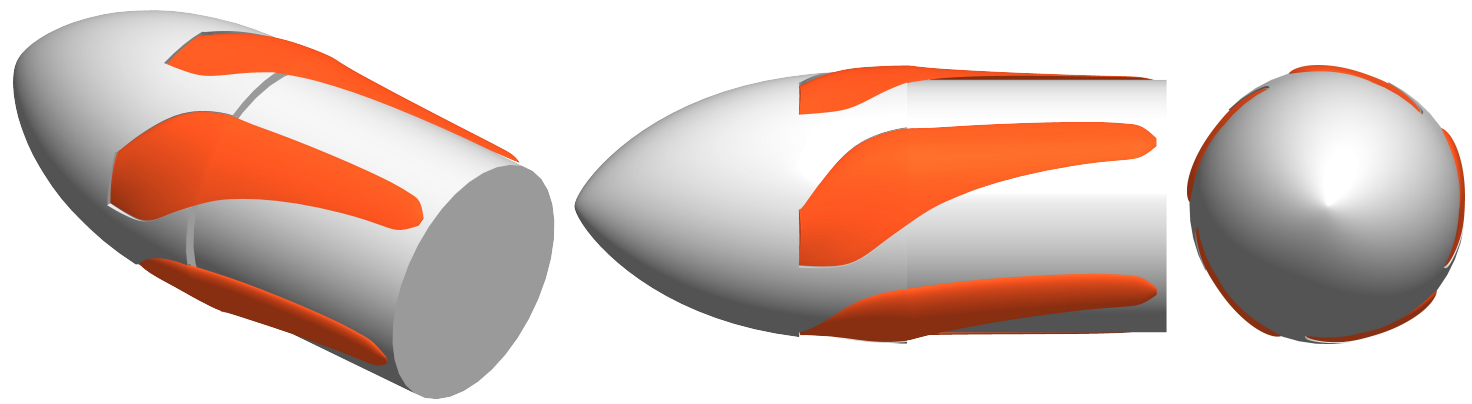

Fig. 5 CAD representation of the larger diameter spinner configuration.

\section{Fully External Blade}

The objective of the fully external blade design is to conform the high-lift propeller blades so that the bottom of the blades skim the nacelle surfaces along the entire blade length, except at the root to ease the flow transition, as shown in Fig. 6. Again, this design does not require that the propeller be stopped at any particular position. The fully external blade design does not result in increased nacelle diameter but does have increased frontal and wetted area from the protruding blades, which will likely increase drag over the recessed version but not as strongly as the larger diameter spinner configuration. This design maintains the maximum nacelle OD of 6.36 inches similar to the fully recessed version.
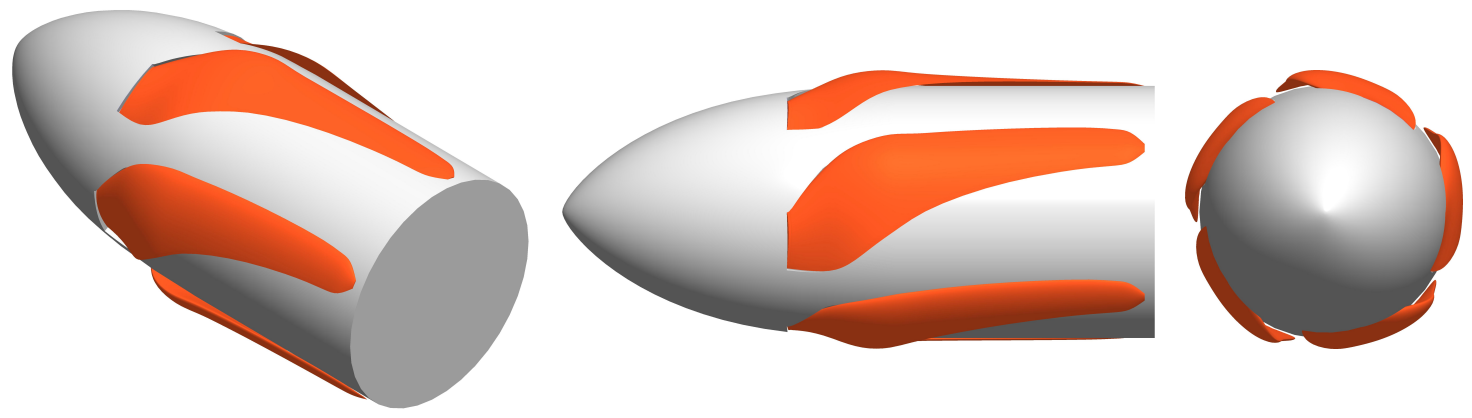

Fig. 6 CAD representation of the fully external blade configuration.

\section{Analysis Method}

\section{A. Analysis Conditions and Models}

The high-lift blade twist and chord distributions and low blade tip speeds do not change between designs; therefore, the results from a simplified blade-element analysis should be identical for all variants. We evaluated each of the designs with OVERFLOW [11, 12]. For the deployed propeller analysis, we used dual time stepping with 20 subiterations and a physical step size corresponding to $1^{\circ}$ of rigid body rotation to predict the time accurate flow field, with at least eight revolutions of propeller travel being simulated. Forces and moments were then averaged over the final revolution. We used the same time stepping scheme and averaging to estimate the stowed blade performance for each design to maintain commonality between the models. Of primary interest were the drag effects of each blade design in the stowed configuration at the X-57 cruise condition. Any significant drag increase would have a negative impact on the X-57's efficiency goals.

To test each design, we installed a propeller and spinner onto a representative wing section, including a wing, pylon, and nacelle, which simplified the analysis. The representative section chord was based on the chord at the most inboard 
nacelle location and the span was defined by the blade diameter with the addition of span on both ends to allow the capture of the propeller streamtube effects on the wing. Figure 7 shows the difference between the OpenVSP models of the deployed and stowed test sections. The CFD test section includes pylon leading edge strakes, which mitigate the formation of pylon leading edge stagnations and vortices; these strakes are not shown in the OpenVSP model.

Stowed configuration analyses were performed with a 150 KTAS freestream velocity at $8000 \mathrm{ft}$ standard altitude and zero angle of attack relative to the propeller axis of rotation. To provide a performance comparison, the fixed-pitch blades were also analyzed at cruise conditions in two additional configurations: fixed in the deployed position and windmilling. Neither of these conditions should be acceptable for operating the high-lift propellers due to the significant expected drag increase and flow disruption over the wing. However, we tested these scenarios to determine the potential magnitude of the detrimental effects.

To determine any performance differences between blade variants, we also examined each deployed configuration at the X-57 high-lift propeller design condition, i.e., a 58 KTAS freestream velocity at sea level standard altitude, zero angle of attack, and the propeller spinning at 4550 RPM ( $450 \mathrm{ft} / \mathrm{s}$ blade tip speed). Prior analyses of conformal high-lift propellers [1] showed a slight decrease in thrust and power for a fixed propeller RPM and we expected to see a similar result in these cases. These analysis cases also determined any difference in the lift augmentation between designs by comparing the lift generated by the blown wing section.

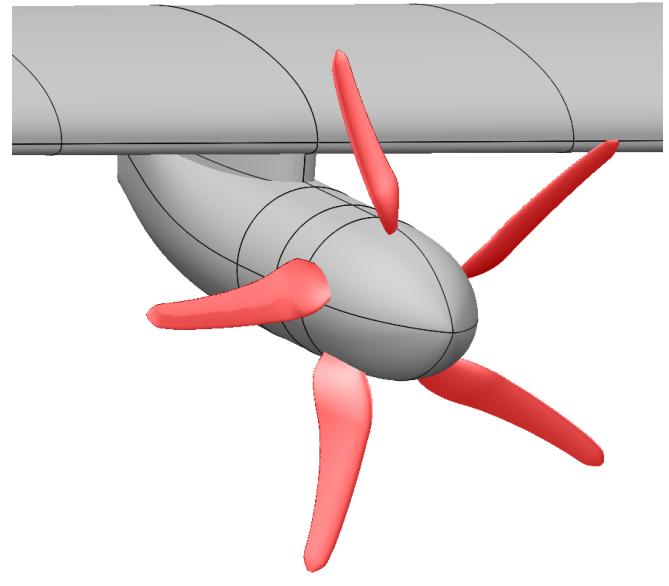

(a) Deployed

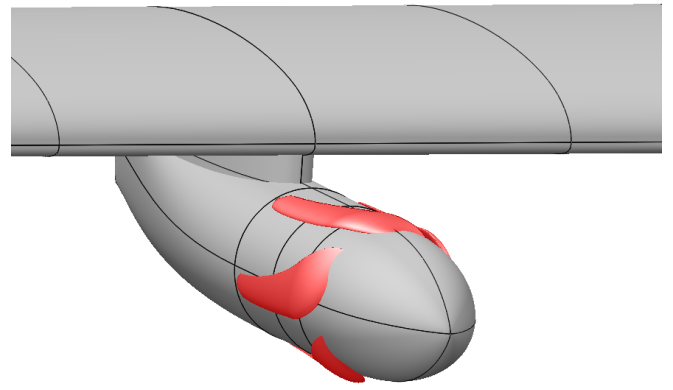

(b) Stowed

Fig. 7 OpenVSP model of the propeller analysis test section with deployed and stowed blades. Pylon leading edge strakes are not shown.

\section{B. Tools and Methods}

\section{XROTOR}

XROTOR is a program for the design and analysis of propellers and windmills that consists of several routines performing functions such as designing minimum induced loss propellers, modifying an existing rotor geometry, twist optimization for minimum loss, and rotor analysis under a variety of input parameters [13]. Other useful functions, such as structural analysis, acoustic analysis, geometry and performance plotting, and slipstream analysis, are also included. Patterson used this tool along with scripts to create the original, constant velocity propeller design for the X-57 high-lift system [10]. We chose to use XROTOR because the software will generate radially distributed chord and twist for a given blade design, which we use as inputs for folding blade algorithms. We also leveraged XROTOR to perform Blade Element Momentum (BEM) analyses of the various high-lift propeller designs in this study; however, because the folding, conformal blade design method intentionally maintains the radial chord and twist distribution of the reference blade, we expected the XROTOR results to be equal among variants. 


\section{OpenVSP}

Open Vehicle Sketch Pad (OpenVSP) is an open-source, parametric geometry-based computer modeling environment originally funded by NASA [14]. The program enables users to rapidly generate a variety of designs using aircraft-native components, such as wings, fuselages, ducts, and propellers. OpenVSP facilitates the initial creation of conceptual models as well as detailed approximations of existing vehicles and objects. The software includes a variety of analysis tools: parasitic drag calculators, inviscid flow solvers, mass property computation, and others. OpenVSP also has the ability to export geometry in a variety of formats such as IGES, Part3D, and STL. We used OpenVSP along with scripts to generate the blade variant and wing test section geometries for analysis and export to other tools.

\section{Chimera Grid Tools}

Chimera Grid Tools (CGT) software contains several pre- and post-processing tools for the Chimera overset approach to gridding for computational fluid dynamics (CFD) analyses on complex geometries [15]. CGT was used to assemble the overset grid systems needed to perform CFD analyses of each design. We used CGT in combination with the EGADS2SRF [16] tool to import STEP file geometries from PTC Creo using a $3 \mathrm{~mm}$ (0.03937 in.) maximum cell edge length.

\section{OVERFLOW}

OVERFLOW (OVERset grid FLOW solver) is a structured, overset grid, Reynolds-Averaged Navier-Stokes, CFD flow solver developed at NASA [11, 12]. A fifth-order WENO5M (Weighted Essentially Non-Oscillatory) method [17] with HLLE++ (Harten, Lax, van Leer and Einfeldt) flux scheme [18] was used to calculate inviscid flux contributions and second-order central differences were used to approximate the viscous flux. A k- $\omega$ SST-RC-QCR2000 (rotation correction with quadratic constitutive relationship) turbulence model $[19,20]$ coupled with the Langtry-Menter CFX-v1.1 transition model [21], despite not being Galilean invariant, was used to maintain commonality with previous X-57 studies [1,2]. This method has been successfully used in other situations with rotational reference frames, e.g., Jain $[22,23]$.

\section{PTC Creo Parametric}

PTC Creo Parametric 3-D modeling software is a computer-aided design (CAD) environment that enables the generation of complex three-dimensional parts and assemblies using surfaces and curves. This software was used to alter the OpenVSP conceptual models by cutting away material from the nacelles, forming solid part representations, and exporting the model in a STEP file format, which was then imported into CGT.

\section{Conformal Blade Design Method}

Our 2017 conformal blade design method paper [1] describes how a stowable propeller can be created such that little to no performance is lost versus a non-folding blade and very little additional drag is generated compared to a clean nacelle. This method restricts the manipulation of the propeller blade sections to only translate within the local blade section plane without altering the local chord or twist. At the low tip speed of the high-lift propellers, this should have no appreciable effect on the propeller's performance because the local blade section velocity remains relatively unchanged. We also supposed that any additional losses from 3-D effects, such as blade tip vortices, would be similar between variants because of the low blade tip speeds and the commonality between blade designs.

In our previous paper on folding blade designs [1], OVERFLOW was used to analyze and compare the performance of non-folding and folding propellers. Table 2 summarizes the results of that study in which the folding propellers were shown to have an approximate $2 \%$ reduction in thrust and average axial velocity and a less than $4 \%$ reduction in torque and power at a fixed propeller RPM. 
Table 2 Comparison of Predicted Propeller Performance for the Non-Folding and Folding-Blade Designs [1].

\begin{tabular}{l|ccc} 
& Original Blade & New Blade & \% Change \\
\hline Thrust (N) & 222 & 217 & $-2.3 \%$ \\
Power (kW) & 10.3 & 9.91 & $-3.8 \%$ \\
Torque (N-m) & 21.5 & 20.8 & $-3.3 \%$ \\
Avg Axial $\Delta V(\mathbf{m} / \mathbf{s})$ & 16.8 & 16.5 & $-1.6 \%$
\end{tabular}

\section{Results}

The results of the stowed and deployed configuration analyses are presented below. Most data are presented in Imperial units while torque and power are presented in metric to be more representative of typical motor performance units.

\section{A. Stowed Configuration Results}

Table 3 summarizes the flow conditions at which the stowed configuration analyses were performed. The results of these analyses, detailed in Table 4, showed very little difference in lift between the clean nacelle and the conformal blade variants. However, the drag did increase as expected across the designs. Flow and pressure visualizations are provided in Fig. 8 to illustrate the conformal blade effects.

Table 3 Flow Conditions and Model References for Stowed Configuration Analysis

\begin{tabular}{ll|ll} 
Parameter & Value & Parameter & Value \\
\hline Freestream Velocity & $150 \mathrm{KTAS}$ & RPM & 0 \\
Altitude & $8000 \mathrm{ft}$ & Reference Area & $6.42012 \mathrm{ft}^{2}$ \\
Angle of Attack & $0^{\circ}$ & Reference Chord & $2.342 \mathrm{ft}$
\end{tabular}

Table 4 Stowed Propeller Configuration Performance

\begin{tabular}{lccccc} 
Configuration & Drag (lbf) & Drag Increase (lbf) & Drag \% Diff & Lift (lbf) & Lift \% Diff \\
\hline Clean Nacelle & 12.14 & 0.00 & $0.00 \%$ & 285.6 & $0.00 \%$ \\
Fully Recessed & 12.28 & 0.14 & $1.14 \%$ & 281.1 & $-1.59 \%$ \\
Fully External & 12.51 & 0.38 & $3.09 \%$ & 284.8 & $-0.28 \%$ \\
Large Spinner & 12.75 & 0.61 & $5.01 \%$ & 284.9 & $-0.25 \%$ \\
\hline Windmilling & 21.96 & 9.82 & $80.88 \%$ & 269.9 & $-5.50 \%$ \\
Fixed (Deployed Position) & 37.92 & 25.78 & $212.36 \%$ & 253.1 & $-11.39 \%$
\end{tabular}

The fully recessed conformal blade design produced the least amount of additional drag at these conditions, only $0.14 \mathrm{lbf}$, and would be the clear winner if drag minimization was paramount. Observing the clean nacelle and fully recessed streamtraces (Figs. 8(a) and 8(b)), we find very little difference in the flow around the nacelle but can see a slightly larger trailing edge "bubble" behind the nacelle tail with the stowed blades. This behavior makes sense due to transitions along the surface between the nacelle and blade causing the flow to become more turbulent. Note that the results indicated an odd artifact where the lift seems to have decreased more than the other configurations over the range of time steps averaged. We suspect that this is due to a small, local pressure disturbance within this time frame that may have a different frequency than the other variants. Running the solution for additional time steps in OVERFLOW should improve the model convergence and remove this artifact. Furthermore, the pressure distribution over the wing upper surface shows almost no discernible difference between the fully recessed configuration and the clean nacelle. Considering the relatively small change in lift, we remain confident in the solution and results shown. 
The fully external blade design produced slightly more additional drag than the fully recessed configuration, $0.38 \mathrm{lbf}$ over the baseline; however, this amount is still small compared to the total test section drag. Examining the streamtraces (Fig. 8(c)), we can see some weak recirculation behind the stowed blade root trailing edge that seems to quickly rejoin the surrounding airflow. The image also shows stagnation points at the blade leading edge where the blade breaks the spinner surface. These stagnation regions did not seem to overly disrupt the surrounding flow though they are likely a contributing source of the additional drag. Again, the lift is essentially unaffected by the design and the pressure distribution over the wing upper surface is very similar to the clean nacelle.

The larger diameter spinner design produced even more drag than either of the other two variants, $0.61 \mathrm{lbf}$ over the baseline, which began to exceed the acceptable amount of additional drag for the configuration. At first glance, the streamtraces in Fig. 8(d) appear to be relatively smooth and similar to the clean nacelle. However, when we placed streamlines in the region directly behind the spinner step, we discovered a significant amount of tightly wrapped recirculation. The flow in these regions also seemed to have difficulty escaping the recirculation and rejoining the surrounding air. These areas likely caused the significant increase in test section drag. The wing upper surface pressure distribution in these results presented an additional concern. Compared to the other models, the pressure is not only slightly increased over this surface but is also quite irregular, which could lead to early flow separation at higher angles of attack.

The fixed-pitch high-lift propellers cannot be feathered to align with the flow. Therefore, we expected extremely high drag increases for the windmilling and fixed-position cases. To find the cruise speed windmilling condition, we iterated the propeller RPM until the resulting torque was very near zero. Eventually, a 4240 RPM propeller speed achieved the desired condition. Unsurprisingly, this configuration added a large amount of drag to the test section, 9.8 lbf or $81 \%$ of the clean nacelle case drag, and thoroughly disrupted the flow over the wing causing a $5.5 \%$ reduction in total lift. The fixed-position condition was even more detrimental. This worst-case condition more than tripled the drag of the clean nacelle baseline and reduced lift by over $10 \%$.

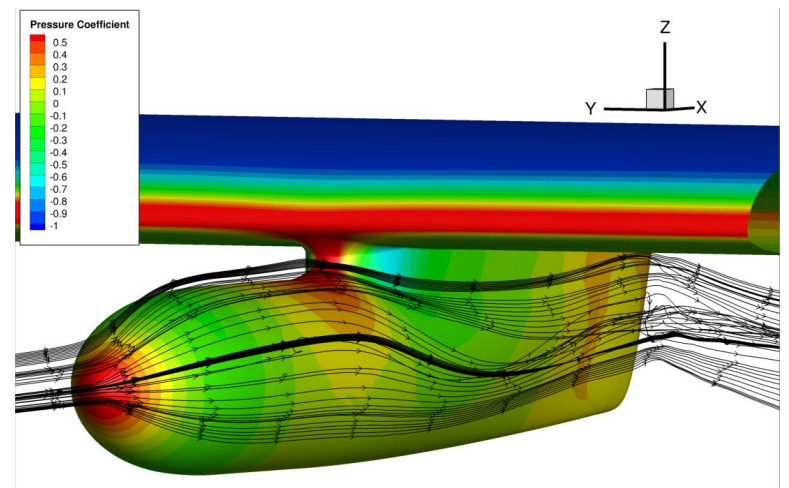

(a) Clean nacelle

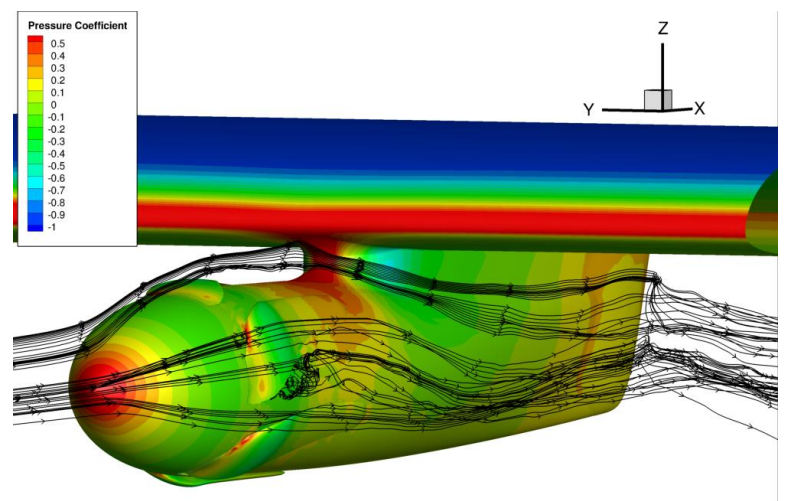

(c) Fully external blades. Weak recirculation behind blade.

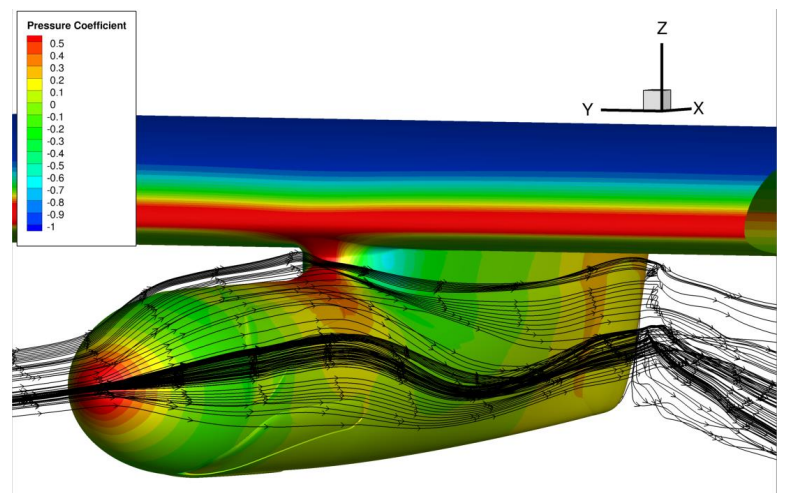

(b) Fully recessed blades

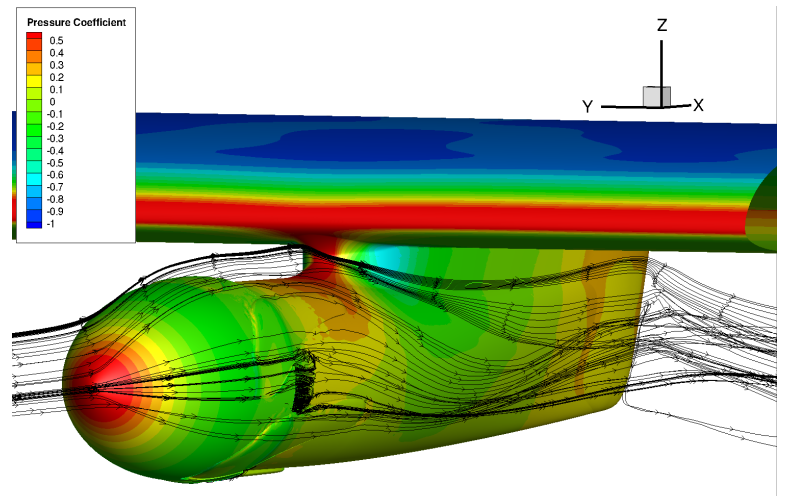

(d) Larger OD spinner. Strong recirculation behind step

Fig. 8 Streamtraces for each of the stowed conditions in comparison to the clean nacelle. 


\section{B. Deployed Configuration Results}

Table 5 describes the flow conditions for the blown, or deployed propeller, configuration analyses. The results of these cases are summarized in Table 6 where we find that the folding, conformal propeller designs perform well compared to a non-conformal baseline. As expected, in each conformal propeller case there was a relatively small reduction in both torque and thrust, on the order of $2 \%$ to $5 \%$ at 4550 RPM. The largest reduction was with the larger spinner configuration where more of the blade root was covered by the spinner. The physical differences between the fully recessed and fully external propellers are quite small when deployed and so it follows that the results show very little performance difference between these two configurations.

Table 5 Flow Conditions and Model References for Deployed Performance Analysis

\begin{tabular}{ll|ll} 
Parameter & Value & Parameter & Value \\
\hline Freestream Velocity & $58 \mathrm{KTAS}$ & $\mathrm{RPM}$ & 4550 \\
Altitude & $0 \mathrm{ft}$ & Reference Area & $6.42012 \mathrm{ft}^{2}$ \\
Angle of Attack & $0^{\circ}$ & Reference Chord & $2.342 \mathrm{ft}$
\end{tabular}

Table 6 Deployed Propeller Performance

\begin{tabular}{lccccccc} 
Configuration & $\begin{array}{c}\text { Thrust } \\
(\mathbf{N})\end{array}$ & $\begin{array}{c}\text { \% Diff: } \\
\text { Thrust }\end{array}$ & $\begin{array}{c}\text { Torque } \\
(\mathbf{N}-\mathbf{m})\end{array}$ & $\begin{array}{c}\text { Power } \\
(\mathbf{k W})\end{array}$ & $\begin{array}{c}\text { \% Diff: } \\
\text { Torque }\end{array}$ & $\begin{array}{c}\text { Lift } \\
\text { (lbf) }\end{array}$ & $\begin{array}{c}\text { \% Diff: } \\
\text { Lift }\end{array}$ \\
\hline Non-conformal & 221.14 & $0.00 \%$ & 22.30 & 10.63 & $0.00 \%$ & 68.98 & $0.00 \%$ \\
Fully Recessed & 215.13 & $-2.72 \%$ & 21.60 & 10.29 & $-3.18 \%$ & 68.13 & $-1.24 \%$ \\
Fully External & 214.08 & $-3.19 \%$ & 21.56 & 10.27 & $-3.33 \%$ & 68.12 & $-1.25 \%$ \\
Larger Spinner & 210.83 & $-4.66 \%$ & 21.16 & 10.08 & $-5.11 \%$ & 68.21 & $-1.12 \%$ \\
\hline Fully External & 222.71 & $0.71 \%$ & 22.30 & 10.63 & $0.00 \%$ & 68.46 & $-0.76 \%$ \\
$\begin{array}{l}\text { Power Matched } \\
\text { (4625 RPM) }\end{array}$ & & & & & & &
\end{tabular}

Fig. 9 shows propeller and nacelle pressure coefficient contours as well as U-velocity slices for all conformal propeller variants along with the non-conformal baseline. The figures illustrate that the induced velocity fields aft of the propeller are very similar across the designs and closely match that of the non-conformal propeller. Interestingly, it seems that the conformal propellers all induced slightly more rotation to the flow aft of the nacelle causing the "bubble" to wrap up into a core.

Similar to the conclusions in 2017, since the true purpose of these propellers is to provide lift augmentation, the slight reductions in performance shown are acceptable to the project, having lift margin built into the design [1]. However, we decided to pursue the hypothesis that matching input shaft power would improve propeller performance such that there would be no discernible difference between non-conformal and conformal designs. We then iterated the fully external input propeller RPM until the torque and power converged to that of the non-conformal baseline. The Fully External Power Matched result in Table 6 predicts that there would be a less than 1\% difference in thrust and lift augmentation compared to the 4550 RPM baseline when the conformal propeller spins at 4625 RPM, or about 75 RPM faster. Considering the conformal propeller's relatively large amount of rake and skew, deviating considerably from the baseline propeller geometry, this result shows that conformal propellers can be designed with minimal performance losses compared to a non-conformal propeller.

Having shown that propeller performance could be matched, we now wished to know the cause of the speed discrepancy. We originally assumed that the blade sweep resulting from the conformal blade profile would have relatively little effect on the overall propeller performance considering the low design tip speed, physically small propellers, and radially distributed flow field. However, we had failed to recognize that the blade sweep was so large relative to the local chord and radius, in some locations as much as $\pm 30^{\circ}$, that the small angle approximation for off-axis translations no longer applied. We now believe that the local blade section velocity is approximately reduced by the cosine of the local sweep as illustrated in Fig. 10 and that the swept blade airfoil sections become slightly misaligned with the flow, causing further issues. Therefore, the propeller RPM must increase to have a relatively equivalent average velocity 
along the blade such that the torque and thrust closely match the unmodified baseline.

The folding blade method could be modified to account for local sweep and design flow and further enhanced to rotate the blade sections in the propeller plane, or about the propeller thrust axis, to achieve a propeller blade design that more closely matches the originally intended induced axial velocity profile. These modifications could potentially remove the need to spin the propeller at higher speeds to achieve the same performance as the non-conformal reference blades. However, because the increase in conformal propeller RPM is relatively small compared to the RPM of the non-conformal reference (about $+1.5 \%$ at the 4550 RPM design speed), changes to the folding method seem unnecessary in this case. This is not to say that other propeller designs would not benefit from the updated method nor that we do not intend to pursue improvements, rather that these changes must be part of a larger future study.

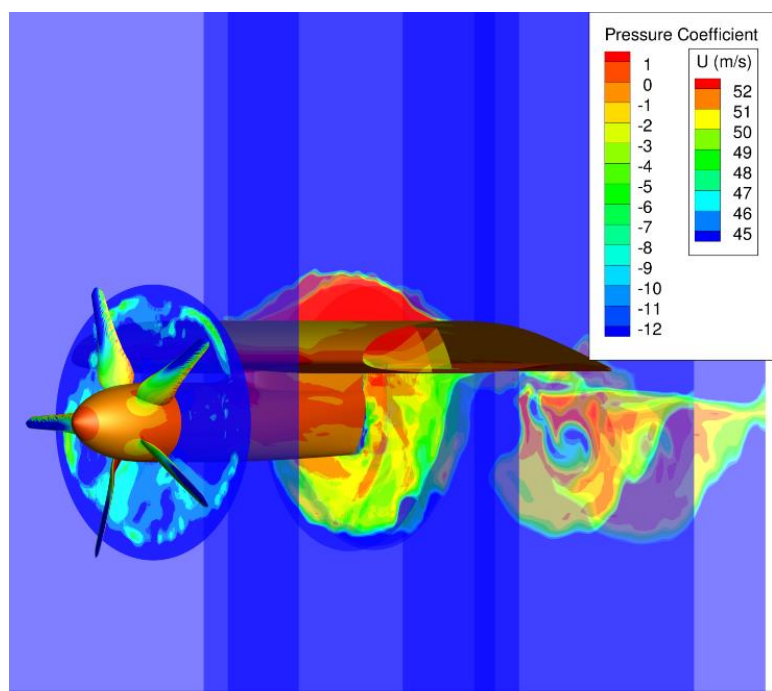

(a) Non-conformal blades

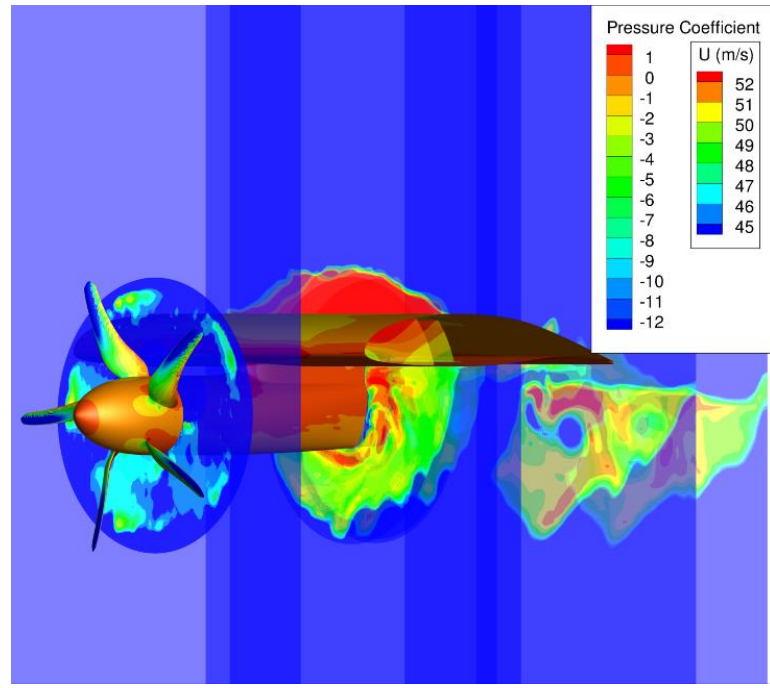

(c) Fully external blades

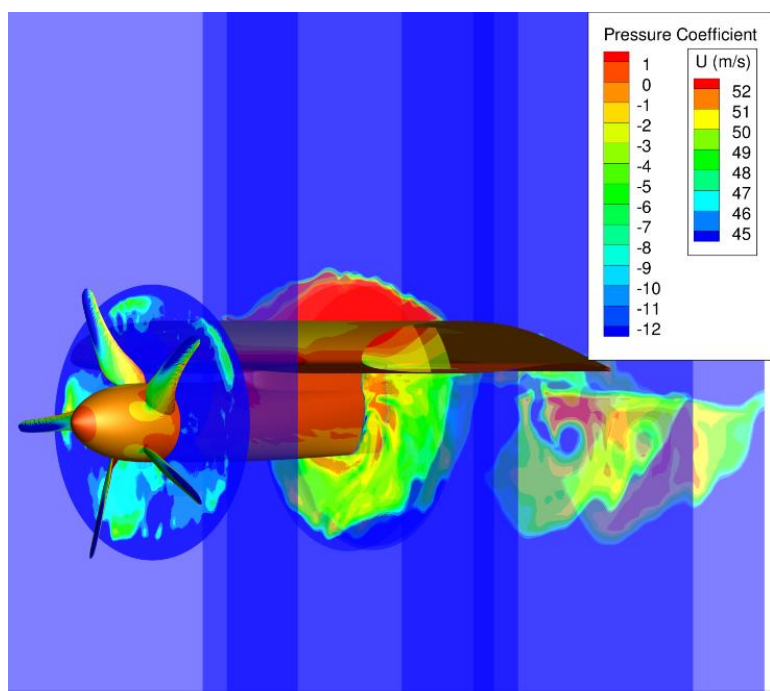

(b) Fully recessed blades

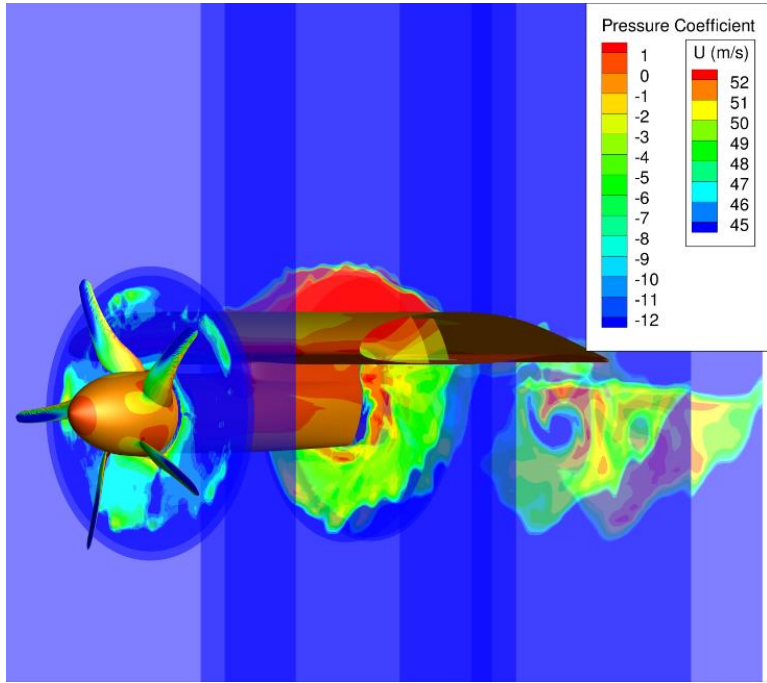

(d) Larger OD spinner

Fig. 9 Pressure contours and $U$ velocity slices along the nacelle for each deployed propeller design. 


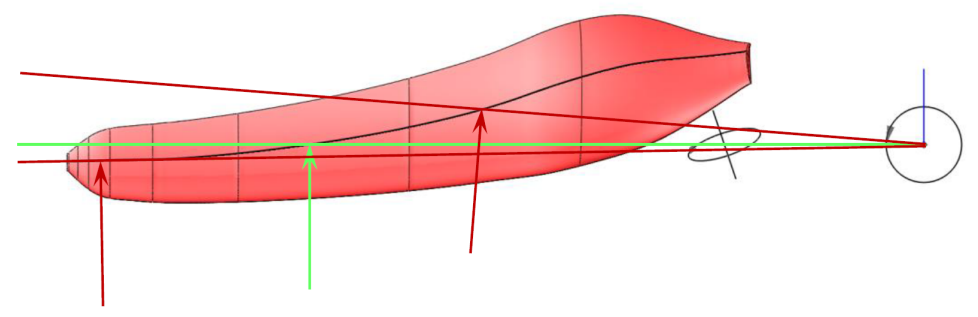

Fig. 10 The blade sweep has a significant cosine reduction effect on the local section velocity. The green axis is the blade radial direction and the red lines indicate off-axis conditions.

\section{Concluding Remarks}

In this study, we have demonstrated that folding, conformal propellers can perform to within $1 \%$ of the design parameters of a non-conforming baseline and that there are several variants of conformal designs that contribute significantly less drag than windmilling or deployed fixed-position configurations. Furthermore, we have established three different methods of conformal design with varying manufacturing and integration complexities that designers may use to determine an acceptable configuration. The fully recessed, minimum-wetted-area design did not perform significantly better than other conformal methods, at least in the case of the X-57 high-lift nacelles.

Although the X-57 design team acknowledges that the minimum-drag-producing variant would be ideal for performance, the manufacturing complexities of such a design may add significant cost and schedule. Therefore, the fully recessed design was traded for another option.

The larger spinner design appeared to be a promising choice. Sacrificing some drag margin for the relatively simple design seemed an acceptable compromise, even with the recirculation aft of the spinner. The remaining nacelle components such as the motor, heat sinks, and interfaces could all be designed as simple, constant diameter cylinders providing significant savings to the X-57 project while only the larger spinner would require cuts to accommodate the blades. However, the larger drag contribution combined with the adverse flow characteristics over the wing lessened the attractiveness of this design.

Therefore, the X-57 design team chose the fully external blade variant to move forward with design, analysis, and production. The relatively small increase in drag versus the clean nacelle and the relative ease of manufacturing and integration provided reductions in cost and schedule while maintaining the efficiency goals of the project.

Future analyses should include angle-of-attack sweeps to examine the effects of each design on stall and flow separation. An extension of the folding blade method and this study would be to examine the efficacy of conformal propellers designed to take advantage of the swept nature of the blades, likely at much higher tip speeds, such as in rotorcraft and vertical takeoff and landing (VTOL) aircraft.

\section{Acknowledgments}

The authors wish to thank NASA's Aeronautics Research Mission Directorate for their support of this effort. The X-57 design effort is currently funded under the X-57 subproject of the Flight Demonstrations and Capabilities Project within the Integrated Aviation Systems Program, after being incubated in the Convergent Aeronautics Solutions Project within the Transformational Aeronautics Concepts Program. Several of the design tools used in this study were largely funded under the Transformational Tools and Technologies Project within the Transformative Aeronautics Concepts Program. Much of the OVERFLOW-related development was funded under the Revolutionary Vertical Lift Project of the Advanced Air Vehicles Program. We would also like to thank Dr. Michael Patterson for his work on designing high-lift propellers and his contribution to the X-57 Maxwell and Dr. Nicholas Borer for his guidance as the X-57 Performance and Sizing IPT Lead.

\section{References}

[1] Litherland, B. L., Patterson, M. D., Derlaga, J. M., and Borer, N. K., "A Method for Designing Conforming Folding Propellers," AIAA AVIATION Forum, American Institute of Aeronautics and Astronautics, 2017. URL https://doi .org/10.2514/6. 
$2017-3781$.

[2] Borer, N. K., Patterson, M. D., Viken, J. K., Moore, M. D., Bevirt, J., Stoll, A. M., and Gibson, A. R., "Design and Performance of the NASA SCEPTOR Distributed Electric Propulsion Flight Demonstrator," 16th AIAA Aviation Technology, Integration, and Operations Conference, AIAA Aviation, American Institute of Aeronautics and Astronautics, 2016. doi:10.2514/6.2016-3920, URL http://dx .doi .org/10.2514/6.2016-3920, aIAA 2016-3920.

[3] Alisport Srl, "Silent 2 Electro: Electric Self-Launch Sailplane FAI CLASS 13.5m,", URL http://www . ali sport . com/eu/ eng/silent2electro.htm, Accessed 18 Oct 2016.

[4] Stemme AG, “S10,”, 2016. URL www. stemme.com, Accessed 18 Oct 2016.

[5] Graupner, "CAM Folding Propeller with Spinner 5 x $2.4(12$ x 6 cm)," https://www.graupnerusa.com/Electric-sailplanefolding-propeller-with-spinner-1335.12.6.html, Accessed 4-10-2017, URL https: //www.graupnerusa.com/Electricsailplane-folding-propeller-with-spinner--1335.12.6.html.

[6] Fredericks, W. J., Moore, M. D., and Busan, R. C., "Benefits of Hybrid-Electric Propulsion to Achieve 4x Cruise Efficiency for a VTOL UAV," 2013 International Powered Lift Conference, AIAA Aviation, American Institute of Aeronautics and Astronautics, 2013. URL http://dx .doi .org/10.2514/6.2013-4324.

[7] Stoll, A. M., "Comparison of CFD and Experimental Results of the LEAPTech Distributed Electric Propulsion Blown Wing," 15th AIAA Aviation Technology, Integration, and Operations Conference, AIAA Aviation, American Institute of Aeronautics and Astronautics, 2015. URL http: //dx. doi .org/10.2514/6.2015-3188, aIAA 2015-3188.

[8] Stoll, A., "Analysis and Full Scale Testing of the Joby S4 Propulsion System," Transformative Vertical Flight Workshop, 2015. URL http://nari.arc.nasa.gov/sites/default/files/attachments/Stoll-TVFW-Aug2015.pdf, http://nari.arc.nasa.gov/sites/default/files/attachments/Stoll-TVFW-Aug2015.pdf, accessed 18 Oct 2016.

[9] Barrell, E., "Folding and Feathering Propeller Test," Yachting Monthly, 2015. URL http://www. yachtingmonthly . com/gear/folding-and-feathering-propeller-test-29807, http:/www.yachtingmonthly.com/gear/folding-andfeathering-propeller-test-29807, accessed 18 Oct 2016.

[10] Patterson, M. D., Borer, N. K., and German, B., "A Simple Method for High-Lift Propeller Conceptual Design," 54th AIAA Aerospace Sciences Meeting, AIAA SciTech, American Institute of Aeronautics and Astronautics, 2016. URL http://dx.doi.org/10.2514/6.2016-0770, aIAA 2016-0770.

[11] Nichols, R., Tramel, R., and Buning, P., "Solver and Turbulence Model Upgrades to OVERFLOW 2 for Unsteady and High-Speed Applications," 24th AIAA Applied Aerodynamics Conference, Fluid Dynamics and Co-located Conferences, San Francisco, CA, 2006. URL http://arc . aiaa.org/doi/abs/10.2514/6.2006-2824, aIAA 2006-2824.

[12] Nichols, R. H., and Buning, P. G., User's Manual for OVERFLOW 2.2, v.2.2m, 2017. URL https://overflow. larc.nasa. gov/home/users-manual-for-overflow-2-2/.

[13] Drela, M., and Youngren, H., XROTOR User Guide, 2003. URL http://web.mit.edu/drela/Public/web/xrotor/.

[14] “OpenVSP,”, URL http: //openvsp.org/, Accessed 18 Oct 2018.

[15] Chan, W. M., Pandya, S. A., Rogers, S. E., Jensen, J. C., Lee, H. C., Kao, D. L., Buning, P. G., Meakin, R. L., Boger, D. A., and Nash, S. M., Chimera Grid Tools User's Manual, v.2.2, 2018. URL https://www.nas.nasa.gov/publications/ software/docs/chimera/index.html.

[16] Chan, W. M., and Pandya, S., "EGADS2SRF Version 1.1,", URL https ://www . nas .nasa.gov/publications/software/ docs/chimera/pages/egads2srf.html, Accessed 18 Oct 2018.

[17] Henrick, A. K., Aslam, T. D., and Powers, J. M., "Mapped weighted essentially non-oscillatory schemes: Achieving optimal order near critical points," Journal of Computational Physics, Vol. 207, No. 2, 2005, pp. 542-567. URL http: //www.sciencedirect.com/science/article/pii/S0021999105000409.

[18] Tramel, R., Nichols, R., and Buning, P., "Addition of Improved Shock-Capturing Schemes to OVERFLOW 2.1," 19th AIAA Computational Fluid Dynamics, Fluid Dynamics and Co-located Conferences, American Institute of Aeronautics and Astronautics, 2009. URL http://dx . doi .org/10.2514/6.2009-3988, aIAA 2009-3988.

[19] Shur, M. L., Strelets, M. K., Travin, A. K., and Spalart, P. R., "Turbulence Modeling in Rotating and Curved Channels: Assessing the Spalart-Shur Correction,” AIAA Journal, Vol. 38, No. 5, 2000, pp. 784-792. URL http: //dx . doi . org/10.2514/2 . 1058. 
[20] Menter, F. R., "Two-equation eddy-viscosity turbulence models for engineering applications," AIAA Journal, Vol. 32, No. 8, 1994, pp. 1598-1605. URL http://dx . doi .org/10.2514/3.12149.

[21] Langtry, R., and Menter, F., “Transition Modeling for General CFD Applications in Aeronautics," 43rd AIAA Aerospace Sciences Meeting and Exhibit, Aerospace Sciences Meetings, American Institute of Aeronautics and Astronautics, 2005. URL http://dx.doi.org/10.2514/6.2005-522.

[22] Jain, R., CFD Performance and Turbulence Transition Predictions on an Installed Model-scale Rotor in Hover, URL https://arc.aiaa.org/doi/abs/10.2514/6.2017-1871.

[23] Jain, R., "Hover Predictions on the S-76 Rotor with Tip Shape Variation Using Helios," Journal of Aircraft, Vol. 55, No. 1, 2018, pp. 66-77. URL https://doi .org/10.2514/1.C034075. 